\title{
COVID-19 Pandemic and Surgical Oncology: Preserving the Academic Mission
}

\author{
Timothy M. Pawlik, MD, $\mathrm{PhD}^{1}$, Douglas S. Tyler, $\mathrm{MD}^{2}$, Baran Sumer, $\mathrm{MD}^{3}$, Funda Meric-Bernstam, MD, $\mathrm{PhD}^{4}$, \\ Ikenna C. Okereke, MD², Joal D. Beane, MD ${ }^{1}$, Priya H. Dedhia, MD ${ }^{1}$, Aslam Ejaz, MD, MPH ${ }^{1}$, \\ Kelly M. McMasters, MD, $\mathrm{PhD}^{5}$, and Kenneth K. Tanabe, $\mathrm{MD}^{6}$ \\ ${ }^{1}$ Department of Surgery, The Ohio State University, Wexner Medical Center, Columbus, OH; ${ }^{2}$ Department of Surgery, \\ University of Texas Medical Branch, Galveston, TX; ${ }^{3}$ Division of Head and Neck Cancer, Department of Otolaryngology, \\ University of Texas Southwestern Medical Center, Dallas, TX; ${ }^{4}$ Department of Investigational Cancer Therapeutics, \\ University of Texas MD Anderson Cancer Center, Houston, TX; ${ }^{5}$ Department of Surgery, University of Louisville School \\ of Medicine, Louisville, KY; ${ }^{6}$ Division of Surgical Oncology, Department of Surgery, Massachusetts General Hospital and \\ Harvard Medical School, Boston, MA
}

\begin{abstract}
Background. The global pandemic of respiratory disease cause by the novel human coronavirus (SARS-CoV-2) has caused untold suffering, loss of life and upheaval in society. The pandemic has lead to massive redirection of health care resources to treat the surge of COVID-19 patients, and enforcement of social distancing to reduce the rate of transmission.
\end{abstract}

Methods. Editorial Board members provided observations of the implications of the pandemic on academic surgical oncology.

Results. Delivery of health care to other populations including cancer patients has been significantly disrupted. The implications both short term and long term threaten preservation of the academic mission in medicine at large, and certainly in the field of surgical oncology.

Conclusions. The effects on surgical oncology training, research and clinical trials are major.

The devastation and destruction brought about by the novel human coronavirus (SARS-CoV-2) pandemic has created daunting challenges to medical centers and

(C) Society of Surgical Oncology 2020

First Received: 23 April 2020;

Published Online: 29 May 2020

K. K. Tanabe, MD

e-mail: ktanabe@partners.org universities. Despite government funding programs to offset hospital losses from the COVID-19 pandemic, the financial impact is enormous and threatens to derail the academic mission. The impact of the COVID-19 pandemic on education and training, research, and conduct of clinical trials is significant and threatens preservation of the academic mission in medicine at large, and certainly in the field of surgical oncology.

\section{FINANCIAL IMPACT}

\section{Surgical Oncologists}

The COVID-19 crisis has affected healthcare systems worldwide, and institutions have introduced extraordinary measures to reduce exposures and limit the mortality from the pandemic. In particular, surgical oncologists have seen a dramatic decrease in operative case numbers. Other services that have a higher proportion of emergent and urgent cases, such as acute care surgery and orthopedic surgery, have continued to operate with a relatively smaller drop in their caseload. The economic impact of this decline will have short- and long-term consequences for the surgical oncologist's practice.

Immediate Effects On 18 March 2020, the Centers for Medicare and Medicaid Services (CMS) issued a recommendation to cancel all elective surgery. ${ }^{1}$ Soon afterward, the American College of Surgeons released a more nuanced list of procedures that should be deferred 
until after the pandemic subsided. ${ }^{2}$ Although it was recommended that many cancer operations proceed as planned, surgical oncologists were expected to experience a temporary reduction of $30-60 \%$ of cases. ${ }^{3}$ Many of these cases will eventually be performed 1-2 months after the pandemic recedes, but there may ultimately be some cases that are never performed. Some patients will choose other definitive treatments (e.g. stereotactic radiation for lung cancer), while other patients will have tumors that become unresectable because of locoregional progression or development of distant metastatic disease. In addition, some patients may decide they no longer want an operation for a variety of reasons. Most surgical oncologists should expect a reduction of approximately $20 \%$ of the operations they would have planned on performing pre-COVID-19, based on data analyzing the effects of delayed surgery on patients with malignancy. ${ }^{4}$

For many large medical centers, the decline in surgical caseload, postponed admissions, and loss of downstream revenue will lead to an immediate loss of hundreds of millions of dollars. ${ }^{5}$ Surgical oncologists have been asked to adapt their practices, including coverage of acute care surgery services and functioning as intensivists, depending on the number of COVID-19 patients in the hospital. When the pandemic subsides, surgical oncologists are likely to be asked to work extended operating room hours during the week and weekend to relieve the backlog of cases. In addition, there may be pressure to broaden the scope of their usual pattern practice to include performing more non-oncologic procedures to help health systems recover at a time when many have implemented hiring freezes as a cost-saving measure.

Long-Term Effects The long-term financial ramifications of the COVID-19 crisis will be felt for years, and the expected consequences to surgical oncologists will be numerous. Some surgical oncologists have already been asked to accept a decrease in their salary this year. Some will also forgo institutional retirement contributions. Scheduled annual pay raises may also be postponed or canceled. For example, the Mayo Clinic implemented a $10 \%$ pay cut to physicians and also announced a hiring freeze. Several other academic centers have recently followed suit by cutting back pay and/or benefits to clinical faculty, including surgeons, in an effort to close budget deficits. Most centers will also likely reduce some personnel positions, such as secretarial staff and advanced practice providers. Other benefits and travel allowances are being curtailed or eliminated for the foreseeable future.

Some surgical oncologists may be asked to change their practice patterns for the next 1-2 years to help with budget deficits. For example, the percentage of non-cancer patients treated by some surgical oncologists may increase as a tactic to load balance instead of hiring additional subspecialists. In addition, surgical oncologists may be asked to expand their referral base to outlying hospitals to increase revenue for the health system. Protected time for research may be temporarily reduced or suspended, as clinical demands may take priority in order to make up for financial shortfalls due to the pandemic.

\section{Healthcare Systems}

Healthcare systems are facing significant financial challenges in addressing the pandemic. Most notable is the abrupt decrease in revenue associated with elective procedures. These procedures include not only screening and surveillance studies such as mammograms, computed tomography (CT) scans, and colonoscopies but also therapeutic cancer operations, especially for early-stage disease. While most of the procedures that have been canceled will be rescheduled once the crisis has started to resolve, there may be persistent lost revenue from surveillance studies that have been postponed from 3 to 6 months.

The decline in elective procedures that has followed as a result of both CMS and state mandates mentioned above has led to a marked decrease in both professional and technical revenue. In addition, there has been a substantial decrease in ambulatory clinic visits and its attendant revenue, with conversion of the majority of patient visits to telehealth visits. Despite some relaxation in the billing requirements for telehealth visits, the amount of revenue generated will not make up for the pre-COVID-19 ambulatory clinic volume. In many healthcare systems, the staff typically utilized to run ambulatory clinics are being redeployed to other areas in need, such as emergency rooms, front-line screening for COVID-19 illness, or inpatient wards. Since the average length of stay for a COVID-19-positive patient who requires hospitalization is about 10 days, ${ }^{6}$ the ability to restart ambulatory clinics may be slowed by the lingering tail effect of patients recovering from COVID-19 illness once the peak new case numbers start to decline. For some free-standing facilities that have a more focused cancer or ambulatory practice, healthcare personnel are paradoxically being laid off or furloughed, as revenue is not available to pay staff who have no place to be redeployed.

There are some financial aspects of the crisis that may help soften the burden. On 27 March 2020, President Trump signed the CARES act to help support healthcare providers fighting the COVID-19 pandemic. This piece of legislation provides US\$100 billion in relief funds to hospitals and other healthcare providers on the front lines of the COVID-19 response by supporting healthcare-related expenses or lost revenue attributable to COVID-19. Given 
that the budget for Medicare was previously US\$750 billion, this new legislation increases the spending by approximately $13 \%$ for this fiscal year. ${ }^{7}$ It also ensures that uninsured Americans can obtain testing and treatment for COVID-19. There was an immediate infusion of US $\$ 30$ billion into the healthcare system starting 10 April 2020 . These payments are not loans and will not need to be repaid. All facilities and providers that received Medicare fee-for-service (FFS) reimbursements in 2019 are eligible for this initial rapid distribution. As a condition to receive these funds, providers must agree not to seek collection of out-of-pocket payments from a COVID-19 patient that are greater than what the patient would have otherwise been required to pay if the care had been provided by an innetwork provider. This quick dispersal of funds will provide relief to both providers in areas heavily impacted by the COVID-19 pandemic and providers who are struggling to keep their doors open due to healthy patients delaying care and canceled elective services. If the facility or provider stopped operating as a result of the COVID-19 pandemic, they are still eligible to receive funds as long as they provided diagnoses, testing, or care for individuals with possible or actual cases of COVID-19. Care does not have to be specific to treating COVID-19, as the Department of Health and Human Services (DHHS) broadly views every patient as a possible case of COVID-19. The payments to providers will be distributed based on the share of total Medicare FFS reimbursements in 2019 as a fraction of the US $\$ 30$ billion pool. ${ }^{8}$

The payment pool will be distributed to providers according to their tax identification number (TIN). Large organizations will receive relief payments based on the number of individual TINs that bill Medicare. Employed physicians should not expect to receive an individual payment directly. Instead the employer organization will receive the relief payment through the billing organization. Individual physicians and providers in a group practice are unlikely to receive individual payments directly, as the group practice will receive the relief fund payment as the billing organization. Finally, solo practitioners who bill Medicare will receive a payment under the TIN used to bill Medicare. $^{8}$

The remaining US $\$ 70$ billion will be used for several initiatives. The DHHS and the Administration are working rapidly on additional targeted distributions to providers that will focus on providers in areas particularly impacted by the COVID-19 outbreak, rural providers, and providers of services with lower shares of Medicare FFS reimbursement, or who predominantly serve the Medicaid population. A portion of the US $\$ 100$ billion Provider Relief Fund will be used to reimburse healthcare providers, at Medicare rates, for COVID-related treatment of the uninsured population that requires diagnosis and/or treatment. As a condition, providers will be obligated to abstain from 'balance billing' any patient for COVID-related treatment. The Families First Coronavirus Response Act will require private insurers to cover an insurance plan member's cost-sharing payment for COVID-19 testing. There have also been commitments secured from private insurers, including Humana, Cigna, UnitedHealth Group, and the Blue Cross Blue Shield system, to waive costsharing payments for treatment related to COVID-19 for plan members. ${ }^{8}$

The CARES legislation also has US $\$ 350$ billion allocated to help small businesses keep workers employed amid the pandemic and economic downturn. This program is the Paycheck Protection Program (PPP), in which the federal government provides $100 \%$ federally guaranteed loans to small businesses. These loans may be forgiven if borrowers maintain their payrolls during the crisis or restore their payrolls afterwards. The loan application process began on 3 April 2020 and ran out of funding on 16 April 2020, following which lawmakers have considered replenishing the program with additional funding. This mechanism would potentially help smaller private practices that have had to delay elective procedures and, as a result, have limited cash flow to maintain personnel and office expenses. The loans can be up to 2.5 times the borrower's average monthly payroll costs but not exceed $\$ 10$ million.

The CARES legislation is different than the CMS Accelerated and Advance Payment Program, which has delivered nearly US $\$ 34$ billion to healthcare providers to help ensure providers and suppliers have the resources needed to combat the pandemic. The CMS accelerated and advance payments are a loan that providers must pay back. The streamlined process implemented by CMS for COVID-19 has reduced processing times for a request of an accelerated or advance payment to under 6 days, down from the previous timeframe of 3-4 weeks. The CMS has received over 25,000 requests from healthcare providers and suppliers for accelerated and advance payments, and have already approved over 17,000 requests. Prior to COVID-19, CMS had approved just over 100 total requests in the past 5 years, with most being tied to natural disasters such as hurricanes. The payments are available to Part A providers, including hospitals, and Part B suppliers, including doctors, non-physician practitioners, and durable medical equipment (DME) suppliers. While most of these providers and suppliers can receive 3 months of their Medicare reimbursements, certain providers can receive up to 6 months of aid. The CMS Accelerated and Advance Payment Program is funded from the Hospital Insurance (Part A) and Supplementary Medical Insurance (Part B) trust funds, which are the same funds used to pay Medicare claims each day. The accelerated and advance payments are a loan that providers must pay back. CMS will begin to 
apply claims payments to offset the accelerated and advance payments 120 days after disbursement. The majority of hospitals, including inpatient acute care hospitals, children's hospitals, certain cancer hospitals, and critical access hospitals will have up to 1 year from the date the accelerated payment was made to repay the balance. All other Part A providers and Part B suppliers will have up to 210 days to complete repayment of accelerated and advance payments, respectively. ${ }^{9}$

These various forms of legislation are probably the first of several to come from the government in an effort to help out. On 21 April 2020, the senate passed a US\$484 billion interim COVID-19 funding bill. This bill will help in three main areas. First, US\$310 billion was earmarked for the PPP mentioned above that ran out of money. Second, US $\$ 60$ billion would go to the small business disaster fund. Finally, US\$100 billion is designated for healthcare, of which US\$75 billion would go to hospitals struggling financially as a result of providing care to COVID-19 patients, and US\$25 billion would support expanded access to testing. ${ }^{10}$

Over the next few months to years, charitable causes will likely serve as a significant revenue stream for healthcare systems. The Bill and Melinda Gates Foundation, as an example, has dedicated US\$125 million toward a 'COVID-19 Therapeutics Accelerator'. Their goal is to facilitate development of a vaccine, accurate testing for coronavirus or antibodies suggestive of previous infection, and manufacturing capability for millions of doses. This foundation, and other philanthropic groups, will have resources available for many healthcare systems. Institutions should seek out potential partnerships to increase their revenue stream while conducting impactful studies on how to combat the COVID-19 pandemic.

While surgical procedures have decreased significantly during the pandemic, the intensive care unit (ICU) census is up in many hospitals. This will lead to increased ICU billings, especially as some hospitals with a large number of ill patients have had to expand the number of ICU beds in their facilities. Many hospitals have coordinated with city officials to open up temporary hospitals, usually converting convention centers or sports facilities into overflow locations. The degree to which such facilities will need to be utilized, as well as the financial impact, remains unclear.

The majority of elective procedures will ultimately be performed at some point in the future, and many organizations are already starting to develop post-COVID-19 recovery plans once the surge of patients with COVID-19 subsides. The Society of Surgical Oncology (SSO) has reviewed recent CMS guidance provided regarding 'Opening Up America Again'. ${ }^{11}$ Post-COVID-19 recovery plans try to take into consideration several factors, such as hospital size, types of patients, and available operating room capacity, to determine the best way to accomplish all of the cases that have been delayed while accommodating normal demand. Physicians and hospitals may achieve a new level of efficiency through rapid implementation of new technologies (e.g. telemedicine) to streamline patient flow through the healthcare system.

\section{COMPLEX GENERAL SURGICAL ONCOLOGY TRAINING}

Training for oncologic surgery requires significant hands-on operative experience, exposure to the perioperative management of oncologic patients, and formal education sessions such as grand rounds and multidisciplinary tumor conferences. Complex General Surgical Oncologic (CGSO) training requires rotating through differing services with distinct patient populations, management strategies, and operative procedures. The current COVID-19 pandemic has had a dramatic effect on these aspects of surgical oncologic training. The demands on hospitals from COVID-19 can adversely impact the ability to perform cancer operations and in many cases require trainees to spend less time on didactics and more time on COVID-19-related clinical care. Exposure to patients and the hospital environment by less experienced surgeons in training can place trainees at increased risk for infection with COVID-19. Surgical oncology programs in regions with a significant burden of COVID-19 patients will see a decline in non-urgent oncologic procedures, as healthcare resources are shifted to meet the challenges of the pandemic. In contrast, programs at hospitals with fewer COVID-19 cases may not be affected as significantly. Urgent cancer cases will still proceed at most institutions, albeit with potential modifications such as preoperative testing for COVID-19 and open rather than laparoscopic operations in some instances. Differences in the incidence of COVID-19 can result in divergent experiences for residents and fellows in surgical training at different institutions. Some of the current challenges facing surgical oncology training and some of the guidelines offered by the Accreditation Council for Graduate Medical Education (ACGME), the American Board of Surgery (ABS), and the CGSO Board (CGSOB) to respond to the COVID-19 pandemic are summarized below.

Although the final treatment decision regarding oncologic surgery remains with the immediate healthcare provider, factors that can influence the decision to delay or to proceed with cancer surgery include the current and projected incidence of COVID-19 in the community, ability to implement telemedicine perioperatively, staffing and supply availability, the health of the individual patient, and the urgency of the procedure. CMS has created a tiered 
framework for the prioritization of care during the COVID19 pandemic. $^{1}$ Similarly, the American College of Surgeons has created a Guidance for Triage of Non-Emergent Surgical Procedures, based on the Elective Surgery Acuity Scale (ESAS). ${ }^{12}$ According to the ESAS, most oncologic operations fall into the Tier 3 category of procedures that cannot be postponed. The volume of Tier 3 procedures is least likely to be affected by the pandemic. However, many institutions have sought to more aggressively utilize or prolong neoadjuvant therapy strategies when possible to delay surgery until after the peak of expected COVID-19 hospitalizations. This will also affect the operative experience of trainees. Lower-risk cancer operations are being postponed or canceled and resident/fellow experience in these lower acuity procedures will be disproportionately decreased. The volume of COVID-19 patients in the specific institution will also have a substantial effect on training case volumes.

The ACGME has defined three stages of operations for sponsoring institutions: Stage 1 is 'Business as Usual'; Stage 2 is increased but manageable clinical demands; and Stage 3, 'crossing a threshold beyond which the increases in the volume of the illness', involves circumstances where routine care and education must be reconfigured significantly. ${ }^{13}$ Stage 1 institutions have no significant disruptions in surgical volumes and have not altered patient care or educational activities. For Stage 1 hospitals, the ACGME has provided flexibility with respect to site visits, selfstudy, and responses to ACGME surveys, but the residency/fellowship training requirements, both common and specialty-specific, remain in effect. ${ }^{14}$ Didactic sessions such as educational conferences and journal clubs should continue, with distance learning implemented as much as possible. Stage 2 institutions have variances from the training program requirements addressed by the 'Stage 2: Increased Clinical Demands Guidance', which stipulates allowable changes to educational programs. ${ }^{15}$ These allowable changes include faculty members being able to provide direct supervision through telemedicine, fellows acting as attending physicians in their core specialty, and flexibility in the management of trainees who may fail to meet educational or graduation requirements due to alterations in clinical responsibilities. Stage 3 institutions are under pandemic emergency status, with most residents and fellows shifting to clinical care and with most, if not all, educational activities suspended. ${ }^{16}$ Only the ACGME Common Program Requirements regarding work hour limits, training, resources, and supervision remain in place. Residents and fellows continue to care for patients under appropriate supervision tailored to the clinical situation and their level of experience. Residents and fellows are provided appropriate training and resources for the range of clinical situations they may encounter, including novel circumstances in this new clinical environment. For example, if new, higher-level personal protective equipment (PPE) is required, residents and fellows need to be provided this equipment and need to be trained in the proper donning and doffing of the PPE. As before, residents and fellows should not work in areas in which they do not have the necessary knowledge or skills. Finally, the work hour limitations for residents/fellows are unchanged.

For Stage 2 and 3 institutions, to address potential shortages in core specialties such as medicine and surgery, fellows in training, and board-eligible or board-certified trainees in their core specialty may be asked by the institution to serve in that role. For example, a board-certified general surgeon completing a CGSO fellowship may be asked to serve as a general surgeon for their institution. This is permitted, as long as this time does not significantly impact their fellowship experience and does not exceed $20 \%$ of their overall time and effort. The ACGME has suspended all other common and specialty-specific program requirements for Stage 3 institutions. ${ }^{16}$

The ABS has modified its training requirements and instituted hardship modifications for surgical training, including CGSO fellowships. ${ }^{17}$ The ABS and CGSO Board are allowing fellows to claim credit for documented educational or clinical time to replace non-voluntary time away from clinical duties. The ABS has also reduced the clinical time and operative case volume requirements by $10 \%$ for the 2019-2020 academic year without the need for documentation. Further absences due to immunocompromised, sick, or pregnant trainees can be submitted as special requests and are evaluated on a case-by-case basis. Missed non-surgical rotations can be made up using acceptable educational alternatives if they are documented and attested to by the program director. The CGSO Board will also not enforce disease-specific site minimums, since fellows may miss one or more specific rotations during the pandemic, as long as the fellows participate in and document acceptable alternative learning tools. These must be approved by the program director and can include CGSO SCORE procedural modules, Annals of Surgical Oncology Landmark Series, and procedural videos or other materials corresponding to the disease site rotation missed, so long as these activities are acceptable to the program director and the Clinical Competency Committee (CCC).

As always, the ACGME and ABS trust program directors and CCCs with the decision on the readiness of residents/fellows for independent practice. This policy was affirmed in a statement of principles sponsored jointly by the ACGME and the American Board of Medical Specialties (ABMS), where the two organizations acknowledge the need for 'flexibility and creativity' to continue maintaining their commitment "to ensure physicians practice medicine safely and efficaciously". To this 
end, they reaffirmed the importance of program directors and CCCs in deciding the readiness for independent practice, especially in "times of crises when traditional timeand volume-based educational standards may be challenged". ${ }^{18}$ Although training may be modified due to the COVID-19 pandemic, if directors institute compensatory educational opportunities, the effects of the pandemic on surgical oncologic training can be minimized.

\section{RESEARCH}

Continuing basic science and translational laboratory research during a pandemic can be particularly challenging. Policies that limit the spread of COVID-19 can directly hamper progress in research. For example, in order to ensure physical distancing, comply with a 'stay-athome' mandate, and preserve PPE supplies, research personnel have limited laboratory access. Select individuals designated by Human Resources who are critical to infrastructure and laboratory maintenance such as animal care are generally allowed to continue work in the laboratory. Furthermore, essential personnel in the same laboratory have utilized alternating work schedules to ensure physical distancing. Experiments are limited to essential research, which has been defined as investigations that impact the immediate health and well-being of patients, such as research related to COVID-19 or research that would endanger lives if discontinued. Importantly, essential research typically does not include critical experiments needed for a high impact paper, experiments that can be performed with physical distancing, experiments needed for a funded grant timeline, or experiments associated with significant cost or time lost if terminated. In addition, laboratory purchasing, shared resources, and core facilities are also restricted to essential research.

With regard to animal colonies, experiments have been discontinued except for approved COVID-19 research activities. Additional access to the vivarium is limited to designated essential personnel responsible for activities related to colony maintenance, such as weaning, cage separation, and genotyping. To promote physical distancing, laboratory members arrange times to work when the animal-care staff are not present in the same room at the same time. Most Institutional Animal Care and Use Committees (IACUC) recognize the state of emergency and have therefore not cited researchers for reducing the frequency of routine monitoring during this period. In some instances, time points have been delayed for long-term animal studies; however, when animal welfare is of concern, researchers have applied for exemptions to conduct work or harvest tissues. In addition to restricting in-person research endeavors, researchers should prepare contingency plans in the event of staff shortages and provide laboratory personnel planning if a member of the laboratory tested positive for SARS-CoV-2.

Some researchers may be able to apply their expertise to help study or provide clinical 'wet-lab' resources for the pandemic. For example, an investigator who studies cancer may be able to study the effects of cancer in conjunction with COVID-19 infection. Investigators who study immunology may turn their focus towards understanding the pathogenesis of the COVID-19 infection. In addition, researchers have directly contributed to clinical laboratories by donating resources such as PPE, sanitizers, reagents for test kits, and other medical supplies.

Due to the pandemic, researchers can experience significant scientific setbacks and stagnation, and also lose sight of the research mission. Several tactics should therefore be entertained to preserve the academic mission. One tactic to cultivate the academic mission during mandated social distancing is the use of tele-mentoring. Formal surgical tele-mentoring is a concept within telemedicine that involves the use of information technology to provide real-time guidance and technical assistance for surgical procedures from an expert physician at a different geographical location. Previous studies have demonstrated no difference in knowledge and skill acquisition when comparing tele-mentoring and onsite mentoring. Telementoring may be particularly important to trainees and younger faculty. In turn, partnering senior faculty with junior faculty and trainees via tele-mentoring can facilitate research and academic productivity, as well as maintain cohesion among faculty. Tele-mentoring/tele-teaching can also help maintain educational curriculum for trainees, be it researchers or clinical trainees, using virtual journal clubs or research presentations.

Another tactic to preserving academic efforts is to identify avenues to pursue alternative academic and research goals while physically separated from laboratory spaces. For example, researchers can continue with analyses of existing data, as well as continue writing grants and manuscripts. Researchers can use time to conduct 'library research' to get (re)acquainted with laboratory and statistical techniques using online resources. Data-based efforts, including health services research, can also be continued via secure servers. Of note, most federal agencies permit charging of expenses to awards (including salaries and benefits paid to employees) when work is performed remotely, or if personnel are redeployed to provide clinical care for COVID-19 patients. ${ }^{19}$ In fact, if unable to work on grant or training activities, salaries and stipends may be charged to National Institutes of Health (NIH) grants. Prior approval is not required to divert faculty from research to clinical work related to COVID-19 until the end of the public health emergency period. The NIH is also allowing 
pre-award costs to be incurred, extensions of post-award reporting, prior approval requirement waivers, and other flexibilities regarding expenditures of funds.

Many institutions have recognized that the COVID-19 pandemic has created circumstances that prevent or interrupt faculty progress toward professional and scholarly achievement. This problem is especially critical for faculty working toward tenure within a limited and specified timeframe. Therefore, institutions should consider offering automatic approval for a 1-year extension of the probationary period to all applicable tenure-track faculty. At Ohio State, eligible tenure-track faculty who opt into the program are automatically granted a 1-year extension to their probationary period (i.e. 'tenure clock'). Faculty who opt in and who have not yet completed their tenure review may choose to move their mandatory review. If faculty opt into the program but later decide that they do not need the additional year in their probationary period, they may request a non-mandatory review following standard policy and procedures.

To further complicate efforts aimed at preserving the academic mission, the COVID-19 pandemic is causing significant financial ramifications to healthcare systems and hospitals. As a result, several institutions have instituted hiring freezes for research staff and faculty, further restricting the academic mission. Decreased revenue streams also negatively impact the availability of institutional research grants and studies not actively supported by existing grants, as the majority of discretional funding has been focused on COVID-19-related efforts. It is critical that medical centers and universities recognize the importance of protecting surgeon-investigators for post-COVID19. Surgeon-scientists, even pre-COVID, were at existential risk from competing clinical and economic pressures. Departments of surgery, with the support of colleges of medicine, medical centers, and the NIH, need to protect and cultivate surgeon-scientists so that this group of important researchers can exist and thrive post-COVID-19. To this end, departments need to identify other potential funding streams, such as endowments and philanthropy, to preserve the surgeon-scientist mission during COVID-19.

\section{CLINICAL TRIALS}

The COVID-19 pandemic has created many challenges for clinical research, given the focus on reducing exposure and preventing transmission of the novel coronavirus. These include (1) protection of research staff and patients on clinical trials; (2) deciding which studies to continue; (3) deciding which patients should be offered clinical trial participation during the pandemic; (4) deciding which patients should continue participation in trials on which they are already enrolled; (5) ensuring appropriate processes are in place for rapidly modifying protocol requirements and addressing waiver requests; and (6) maintaining oversight of clinical trials to ensure safety and greatest scientific benefit.

\section{Safety}

One of the most important considerations during the COVID-19 pandemic is ensuring safety for research staff and patients on clinical trials. For patient safety, primary strategies include reducing the number of visits, procedures, and sample collections without compromise to primary endpoints or patient safety. These strategies are generally aligned with overall institutional goals of decreasing non-essential patient visits, reducing numbers of elective operations and inpatients, reducing use of ICUs for non-COVID patients, and conservation of PPEs. Secondary endpoints, especially correlatives, may be compromised as a result of these adaptations. Telehealth platforms are often readily substituted for in-person visits, with approval from the Institutional Review Board (IRB).

\section{Maintaining Specific Clinical Trials}

Institutions have been faced with the challenge of making determinations on which clinical trials to keep open amid redirection of institutional goals towards social distancing, reducing the frequency of procedures, and preserving PPEs. Studies that require frequent in-person interactions (hospital visits, laboratory draws, imaging) have generally stopped enrolling new patients onto the study, although this is a case-by-case decision dependent on the nature of the study, associated risks, and the availability of alternative standard-of-care treatment options. As an example of a specific challenge, the COVID-19 pandemic complicates timing and sequence of treatments; patient participation in clinical trials of neoadjuvant therapy, where the timing of surgical procedures is critical to study endpoints, are particularly complicated during the COVID-19 pandemic. Multiple major deviations may lead to a failed clinical trial, or worse, erroneous conclusions. Accordingly, as challenges mount, a clinical trial may need to be temporarily suspended. ${ }^{20}$ Non-therapeutic clinical trials have generally been put on hold in most institutions. Protocols that do not require in-person visits may continue to enroll patients without concern, yet accrual may be reduced due to the economic impact of the pandemic or resource limitations. Decisions to continue clinical trials or put them on hold are made in conjunction with the Office of Responsible Research Practices, the IRB, and Independent Ethics Committees. These institutional bodies are informed by national recommendations from the US FDA 
Guidance on Conduct of Clinical Trials of Medical Products during COVID-19 Public Health Emergency: Guidance of Industry, Investigators, and Institutional Review Boards. ${ }^{21}$

For patients with advanced disease, delay of therapy (most commonly systemic therapy) could have dire consequences and is generally not an option. In this patient population, the additional risk of community COVID-19 transmission associated with clinical trial participation is generally outweighed by the risk of not treating the cancer.

\section{Treatment Selection}

Prior to the pandemic, cancer treatment recommendations routinely involved consideration of clinical trial participation. Amidst the pandemic, these recommendations necessarily balance exposure to COVID-19 against potential benefit to the patient. In scenarios of true equipoise between standard of care and a particular clinical trial, the challenges associated with the conduct of the clinical trial may appropriately result in favoring standard of care. In contrast, enrollment on a clinical trial may be considered in spite of the undergoing COVID-19 pandemic when the therapeutic trial has reasonable likelihood of conferring benefit, may be the superior option, or there are no meaningful standard-of-care options.

In contrast, studies such as window of opportunity trials that explore the effect of therapies given for a short course prior to surgery, although scientifically important, may not meet the threshold of clinical benefit to warrant continuation during the COVID-19 pandemic. When considering therapeutic trials with systemic therapy, the adverse effect profile of the therapeutic agent and potential adverse effects should also be considered to minimize the risk of immunosuppression.

\section{Patient Selection}

An important consideration when considering an individual patient for a specific clinical trial is whether the patient is able to comply with the logistical challenges. Although these considerations were already important in patient selection prior to the pandemic, the challenges have been compounded during the pandemic. Thus, the frequency of in-person visits, mode of transportation to travel to the clinical trial center, and patient independence are necessary considerations in assessing anticipated compliance with the study calendar. The need for social distancing and limitations placed on visitors who may accompany patients add challenges for clinical trial patients.

\section{Trial Logistics}

Given the risks and challenges associated with patient travel combined with institutional goals of reducing nonessential patient visits, procedures, and visitors, changes in clinical trial management are required. Protocol modifications typically require an amendment and a multistep, lengthy review process; however, these requirements have been waived at many centers when these criteria are met: (1) the modifications involve postponing or changing inperson research activities to be conducted remotely; (2) the modifications will only last for the duration of the COVID19 outbreak; and (3) the protocol changes do not pose a significant increased risk to the patient. A thorough reappraisal of the resources available to conduct a clinical trial should be made frequently. Among others, one must reassess whether new safety risks are present to staff and participants, the availability of research staff, and the availability of the intervention.

Given the current challenges, there will likely be increased protocol deviations, including forgoing nonessential sample collections, delayed procedures, and missing data at some time points. The key focuses are on maintaining patient and study staff safety while maximizing the likelihood of therapeutic benefit and obtaining important scientific information. The mandate to decrease elective procedures such as biopsies, imaging, and operations is expected to lead to many protocol deviations, although the expectation remains to comply with trial protocols as closely as possible while aligning with COVID-19-related safety restrictions. All deviances with the protocol should be tracked carefully, as well as documented and reported; there should be no accommodations made for meeting this minimum standard. The 'Stay Home Work Safe' approach still allows for clinical research staff to work remotely, file amendments, monitor and track protocol compliance, and file reports as necessary. Institutions will necessarily take into consideration the impact of the COVID-19 pandemic in formulating responses to these reports.

Strategies to maintain patients on clinical trials include having patients seen by local non-research affiliated providers, shipping oral investigational therapies, and use of non-research affiliated facilities for scans. Increasing the availability of telemedicine further enhances the capability of managing patients from a distance, with the caveat that states differ widely in their acceptance of telemedicine. Unique challenges include developing workflows for issues such as pill counts, as well as processes for remote consenting. Ultimately the skills that the research community gain during this crisis are likely to make us stronger in years to come. 


\section{CONCLUSIONS}

In summary, the COVID-19 pandemic has caused loss of life, great suffering, turmoil in society, and major disruptions in healthcare delivery. The financial impact on hospitals and universities, biomedical research, and education will be felt for years to come. Despite the broad impact of the pandemic, surgeons can maintain their focus on creating novel strategies to mitigate the impact of COVID-19 in order to advance the field of surgical oncology.

DISCLOSURES Dr. Funda Meric-Bernstam reports receiving grant and financial support from Aileron Therapeutics, Inc., AstraZeneca, Bayer Healthcare Pharmaceutical, Calithera Biosciences Inc., Curis Inc., CytomX Therapeutics Inc., Daiichi Sankyo Co. Ltd., Debiopharm International, eFFECTOR Therapeutics, Genentech Inc., Guardant Health Inc., Millennium Pharmaceuticals Inc., Novartis, Puma Biotechnology Inc., and Taiho Pharmaceutical Co., in addition to consulting/advisory board relationships with Aduro BioTech Inc., DebioPharm, eFFECTOR Therapeutics, F. Hoffman-La Roche Ltd, Genentech Inc., IBM Watson, Jackson Laboratory, Kolon Life Science, OrigiMed, PACT Pharma, Parexel International, Pfizer Inc., Samsung Bioepis, Seattle Genetics Inc., Tyra Biosciences, Xencor, Zymeworks, Immunomedics, Inflection Biosciences, Mersana Therapeutics, Puma Biotechnology Inc., Seattle Genetics, Silverback Therapeutics, and Spectrum Pharmaceuticals. Timothy M. Pawlik, Douglas S. Tyler, Baran Sumer, Ikenna C. Okereke, Joal D. Beane, Priya H. Dedhia, Aslam Ejaz, Kelly M. McMasters, and Kenneth K. Tanabe have no conflicts to disclose.

\section{REFERENCES}

1. Centers for Medicare and Medicaid Services. Non-emergent, elective medical services, and treatment recommendations. 2020. Available at: https://www.cms.gov/files/document/cms-non-eme rgent-elective-medical-recommendations.pdf.

2. American College of Surgeons. COVID-19: elective case triage guidelines for surgical care. 2020. Available at: https://www.facs. org/covid-19/clinical-guidance/elective-case.

3. Sathya C. Your elective surgery will be canceled. It's for everyone's good. Washington: The Washington Post; 2020.

4. Pergialiotis V, Haidopoulos D, Tzortzis AS, Antonopoulos I, Thomakos N, Rodolakis A. The impact of waiting intervals on survival outcomes of patients with endometrial cancer: a systematic review of the literature. Eur J Obstet Gynecol Reprod Biol. 2020; 246:1-6.

5. Braithwaite S, Friedman B, Mutter R, Handrigan M. Microsimulation of financial impact of demand surge on hospitals: the H1N1 influenza pandemic of fall 2009. Health Serv Res. 2013; 48(2 Pt 2):735-52.

6. Wu J, Liu J, Zhao X, Liu C, Wang W, Wang D, et al. Clinical characteristics of imported cases of COVID-19 in Jiangsu Province: amulticenter descriptive study. Clin Infect Dis. Epub 29 Feb 2020. https://doi.org/10.1093/cid/ciaa199.

7. Centers for Medicare and Medicaid Services. NHE Fact Sheet. 2020. Available at: https://www.cms.gov/Research-Statistics-Da ta-and-Systems/Statistics-Trends-and-Reports/NationalHealthEx pendData/NHE-Fact-Sheet.
8. US Department of Health and Human Services. CARES Act Provider Relief Fund. 2020. Available at: https://www.hhs.gov/ provider-relief/index.html.

9. Centers for Medicare and Medicaid Services. CMS approves approximately $\$ 34$ Billion for providers with the accelerated/ advance payment program for medicare providers in one week. 2020. Available at: https://www.cms.gov/newsroom/press-release s/cms-approves-approximately-34-billion-providers-accelerateda dvance-payment-program-medicare.

10. Carney J. Senate passes $\$ 484 \mathrm{~B}$ coronavirus relief package. The Hill. 2020. Available at: https://thehill.com/homenews/senate/49 3959-senate-passes-484b-coronavirus-relief-package.

11. Howe JR, Bartlett DL, Tyler DS, et al. COVID-19 guideline modifications as CMS announces "Opening Up America Again." Ann Surg Oncol. 2020. https://doi.org/10.1245/s10434-020-0856 5-9.

12. American College of Surgeons. COVID-19: guidance for triage of non-emergent surgical procedures. 2020. Available at: https:// www.facs.org/covid-19/clinical-guidance/triage.

13. Accreditation Council for Graduate Medical Education. ACGME response to pandemic crisis. 2020. [cited 14 April 2020]. Available at: https://acgme.org/covid-19.

14. Accreditation Council for Graduate Medical Education. Three stages of GME during the COVID-19 Pandemic. 2020. Available at: https://acgme.org/COVID-19/Three-Stages-of-GME-During-t he-COVID-19-Pandemic.

15. Accreditation Council for Graduate Medical Education. Stage 2: increased clinical demands guidance. 2020. Available at: http s://acgme.org/COVID-19/Stage-2-Increased-Clinical-DemandsGuidance.

16. Accreditation Council for Graduate Medical Education. Stage 3: pandemic emergency status guidance 2020 [cited 14 April 2020]. Available at: https://acgme.org/COVID-19/Stage-3-Pandemic-E mergency-Status-Guidance.

17. American Board of Surgery. Modifications to training requirements. 2020. Available at: http://www.absurgery.org/default. jsp?news_covid19_trainingreq.

18. Accreditation Council for Graduate Medical Education. The American Board of Medical Specialties and Accreditation Council for Graduate Medical Education Joint Principles: Physician Training During the Coronavirus Disease 2019 Pandemic. 2020. Available at: https://acgme.org/Newsroom $/ \mathrm{Ne}$ wsroom-Details/ArticleID/10192/The-American-Board-of-Medic al-Specialties-and-Accreditation-Council-for-Graduate-MedicalEducation-Joint-Principles-Physician-Training-During-the-Cor onavirus-Disease-2019-Pandemic.

19. Council on Government Relations. COGR's Federal Agency Guidance on COVID-19's Impact on Proposals and Awards-V1. 2020. Available at: https://www.cogr.edu/sites/default/files/COG R\%20FAQs\%20on\%20COVID-19\%20Impact\%20on\%20Federal $\% 20$ Awards $\% 20$ V.1.1.pdf.

20. Moore TJ, Zhang H, Anderson G, Alexander GC. Estimated costs of pivotal trials for novel therapeutic agents approved by the US Food and Drug Administration, 2015-2016. JAMA Intern Med. 2018; 178(11):1451-7.

21. Food and Drug Administration. FDA guidance on conduct of clinical trials of medical products during COVID-19 Public Health Emergency. 2020. Available at: https://www.fda.gov/med ia/136238/download.

Publisher's Note Springer Nature remains neutral with regard to jurisdictional claims in published maps and institutional affiliations. 